\title{
Single-atom quantum memory with degenerate atomic levels
}

\author{
V. A. Reshetov, E. N. Popov \\ Department of General and Theoretical Physics, Tolyatti State University, 14 \\ Belorousskaya Street, 446667 Tolyatti, Russia
}

\begin{abstract}
The storage and retrieval of a single-photon polarization q-bit by means of STIRAP through the atoms with degenerate levels is studied theoretically for arbitrary polarization of the driving laser field and arbitrary values of the angular momenta of resonant atomic levels. The dependence of the probability of long-term photon storage on the polarization of the driving field and on the initial atomic state is examined.
\end{abstract}

\section{Introduction}

The search for an efficient optical quantum memory and its implementation for quantum networks is an active area (see, e.g., the reviews [1, 2, 3, 4]). The role of carrier of information - flying q-bit - in such devices is played by a single photon and the most natural way to encode the q-bit is provided by its two polarization degrees of freedom (see [5, 6, 7, 8, for some recent proposals). While in the majority of the proposed schemes the photon quantum state is mapped to the quantum state of some atomic ensemble [6, 7, 8, in the experiment [5] the photon polarization q-bit was recorded in the superposition of magnetic substates of the long-lived degenerate level of a single rubidium atom. Such single-atom quantum memory offers an advantage of processing of the stored quantum information. Since at the storage stage the state of a single atom, in which the photon q-bit is encoded, is well determined, it may be altered in a controlled way, e.g., by some laser pulse, so that the state of the retrieved photon will differ from that of the recorded one in a desirable way implementing thus the performance of quantum gates. The setup employed in [5] for the photon storage and retrieval was based on the same technique of vacuum stimulated Raman scattering involving adiabatic passage (STIRAP), which was previously successfully employed for deterministic single photon emission [9, 10, 11. In these experiments the three-level $\Lambda$ - type atom was trapped inside the highfinesse cavity, one branch of the atomic $\Lambda$ - type transitions was coupled to the quantized cavity field, while the other one was coupled to the driving coherent laser field. The STIRAP with the three-level atom with non-degenerate levels is well described in the reviews and textbooks (see, e.g., [12, 13]). In case of non-degenerate levels there exists the only dark state - the superposition of the ground state $a$ and some metastable state $b$ - uncoupled to the excited level $c$ (Figure 1). In course of Raman scattering the atom is adiabatically transferred 


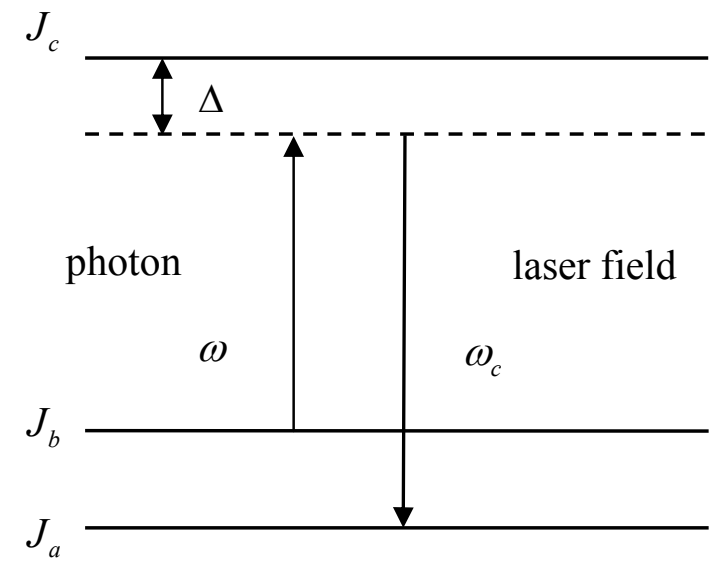

Figure 1: The level diagram

from the initial state $b$ to the target state $a$. The STIRAP with degenerate atomic levels and with classical coherent resonant fields was studied in 14, were it was shown that unlike the non-degenerate case the population from the initial state $b$ is not always totally transferred to the target level $a$. The objective of the present paper is to analyze the process of storage and retrieval of a single-photon polarization q-bit by means of STIRAP through the atoms with degenerate levels for arbitrary values of the angular momenta of resonant atomic levels and to study the dependence of the probability of long-term photon storage on the polarization of the driving field and on the initial atomic state.

In section 2 the interaction model is described and the instantaneous eigenvectors of the interaction operator, which determine the evolution operator in the adiabatic approximation, are constructed. In case of degenerate levels there appear the new types of these eigenvectors, non-existing in case of nondegenerate levels, like the dark states, which atomic part belongs to only one of the lower levels $a$ or $b$, and the bright states, which couple the excited level $c$ with only one of the lower levels $a$ or $b$. In section 3 the formula for the probability of long-term storage of the photon polarization q-bit is obtained and the conditions for the photon storage with unit probability independent on its polarization are outlined. The transitions with the angular momenta $J_{b}=J_{c}=1$, $J_{a}=2$, corresponding to the transitions between the hyperfine structure components of the electronic levels $5^{2} S_{1 / 2}$ and $5^{2} P_{1 / 2}$ of the ${ }^{87} R b$ atom, which were employed in the experiments [5], are analyzed.

\section{Basic equations}

We consider the three-level $\Lambda$ - type atom in a high-finesse cavity, one branch the transition $J_{a} \rightarrow J_{c}$ with the frequency $\omega_{c 0}$ is coupled to the driving coherent laser field, while the other - the transition $J_{b} \rightarrow J_{c}$ with the frequency $\omega_{0}$ is 
coupled to the quantized cavity field (Figure 1 ), $J_{a}, J_{b}$ and $J_{c}$ being the values of the angular momenta of the levels. The electric field strength of the coherent laser field may be put down as follows:

$$
\mathbf{E}_{c}=e_{c}(t) \mathbf{l}_{c} e^{-i \omega_{c} t}+\text { c.c. }
$$

while the quantized field of the cavity in the interaction representation is described by the operator:

$$
\hat{\mathbf{E}}=e(t)\left(\hat{a}_{1} \mathbf{l}_{1}+\hat{a}_{2} \mathbf{l}_{2}\right) e^{-i \omega t}+\text { h.c. }
$$

where the carrier frequencies $\omega_{c}$ and $\omega$ are in resonance with the frequencies $\omega_{c 0}$ and $\omega_{0}, e_{c}(t)$ and $\mathbf{l}_{c}$ are the slowly varying amplitude and the unit polarization vector of the driving field, $e(t)$ is the slowly varying amplitude of the photon field, $\mathbf{l}_{1}$ and $\mathbf{l}_{2}$ are the two unit orthogonal vectors of the two polarization modes of the photon field, $\hat{a}_{1}$ and $\hat{a}_{2}$ are the photon annihilation operators for this modes. The temporal dependence $e(t)$ of the cavity field amplitude appears due to some tailored alteration of cavity parameters. The equation for the slowly-varying density matrix $\hat{\rho}$ of the system, which consists of a single three-level atom and two-mode cavity field, in the rotating-wave approximation and in case of Raman resonance $\omega_{c 0}-\omega_{c}=\omega_{0}-\omega=\Delta$ is as follows:

$$
\begin{gathered}
\frac{d}{d t} \hat{\rho}=\frac{i}{2}[\hat{V}(t), \hat{\rho}], \\
\hat{V}(t)=-2 \Delta \hat{P}_{c}+\hat{G}(t)+\hat{G}^{\dagger}(t), \\
\hat{G}(t)=\Omega_{a}(t) \hat{g}_{a}+\Omega_{b}(t) \hat{g}_{b}, \\
\hat{g}_{b}=\hat{g}_{b 1} \hat{a}_{1}^{\dagger}+\hat{g}_{b 2} \hat{a}_{2}^{\dagger} .
\end{gathered}
$$

Here $\hat{P}_{c}$ is the projector on the subspace of the atomic excited level $J_{c}, \Omega_{a}(t)=$ $2\left|d_{a}\right| e_{c}(t) / \hbar$ and $\Omega_{b}(t)=2\left|d_{b}\right| e(t) / \hbar$ are the reduced Rabi frequencies for the coherent laser field and for the cavity field, $d_{a}=d\left(J_{a} J_{c}\right)$ and $d_{b}=d\left(J_{b} J_{c}\right)$ are the reduced matrix elements of the electric dipole moment operator for the transitions $J_{a} \rightarrow J_{c}$ and $J_{b} \rightarrow J_{c}$, while

$$
\hat{g}_{a}=\hat{\mathbf{g}}_{a} \mathbf{l}_{c}^{*}, \hat{g}_{b i}=\hat{\mathbf{g}}_{b} \mathbf{l}_{i}^{*}, i=1,2,
$$

$\hat{\mathbf{g}}_{a}$ and $\hat{\mathbf{g}}_{b}$ are the dimensionless electric dipole moment operators for the transitions $J_{a} \rightarrow J_{c}$ and $J_{b} \rightarrow J_{c}$. The matrix elements of the circular components $\hat{g}_{\alpha q}(\alpha=a, b ; q=0, \pm 1)$ of these vector operators are expressed through Wigner 3J-symbols [15]:

$$
\left(\hat{g}_{\alpha q}\right)_{m_{\alpha}, m_{c}}=(-1)^{J_{\alpha}-m_{\alpha}}\left(\begin{array}{ccc}
J_{\alpha} & 1 & J_{c} \\
-m_{\alpha} & q & m_{c}
\end{array}\right),
$$

The solution of the equation (1) is expressed through the evolution operator $\hat{S}(t)$ :

$$
\hat{\rho}(t)=\hat{S}(t) \hat{\rho}(0) \hat{S}^{+}(t)
$$


In the adiabatic approximation the evolution operator $\hat{S}(t)$ is defined by the instantaneous eigenvectors $\mid v_{k}(t)>$ and eigenvalues $\lambda_{k}(t)$ of the interaction operator $\hat{V}(t)$ in a following way:

$$
\begin{gathered}
\hat{S}(t)=\sum_{k} \exp \left\{i \phi_{k}(t)\right\}\left|v_{k}(t)><v_{k}(0)\right|, \\
\phi_{k}(t)=\frac{1}{2} \int_{0}^{t} \lambda_{k}\left(t^{\prime}\right) d t^{\prime}
\end{gathered}
$$

Since only single photon storage and retrieval are discussed in the present paper and no decay or dephasing processes are taken into account it is sufficient to limit the system space to the subspace with the basis vectors $\left|J_{a} m_{a}>\right| 0,0>$, $\left|J_{b} m_{b}>\right| 1,0>,\left|J_{b} m_{b}>\right| 0,1>$ and $\left|J_{c} m_{c}>\right| 0,0>$, where $\left.\left|J_{a} m_{a}>,\right| J_{b} m_{b}\right\rangle$ and $\mid J_{c} m_{c}>$ denote the atomic Zeeman states, while $\mid n_{1}, n_{2}>\left(n_{1,2}=0,1\right)$ are the field number states with $n_{1}$ photons in the first polarization mode and $n_{2}$ - in the second. This subspace with the dimension $N=2\left(J_{a}+2 J_{b}+J_{c}+2\right)$ constitutes the invariant subspace of the interaction operator $\hat{V}(t)$, so that its matrix represents itself a square hermitian $N \times N$ matrix. The states $\mid a>$ $\mid 0,0>$ and $|c>| 0,0>$, which atomic part belongs to the level $a$ or $c$, may be represented as columns with $2 J_{a}+1$ or $2 J_{c}+1$ elements correspondingly, while the states $\left|b_{1}>\right| 1,0>+\left|b_{2}>\right| 0,1>$, which atomic part belongs to the level $b$, may be represented as columns with $2\left(2 J_{b}+1\right)$ elements:

$$
\left|b_{1}>\right| 1,0>+\left|b_{2}>\right| 0,1>=\left(\begin{array}{l}
\mid b_{1}> \\
\mid b_{2}>
\end{array}\right) \text {. }
$$

Then the operator $\hat{g}_{a}$ will be represented by the $\left(2 J_{a}+1\right) \times\left(2 J_{c}+1\right)$ matrix, while the operator $\hat{g}_{b}$ will be represented by the $2\left(2 J_{b}+1\right) \times\left(2 J_{c}+1\right)$ matrix

$$
\hat{g}_{b}=\left(\begin{array}{c}
\hat{g}_{b 1} \\
\hat{g}_{b 2}
\end{array}\right)
$$

were each block $\hat{g}_{b i}(i=1,2)$ represents itself a $\left(2 J_{b}+1\right) \times\left(2 J_{c}+1\right)$ matrix.

In order to find out the instantaneous eigenvectors $\mid v_{k}(t)>$ and eigenvalues $\lambda_{k}(t)$ of the interaction operator $\hat{V}(t)$ let us start with the operator

$$
\hat{G}^{\dagger}(t) \hat{G}(t)=\Omega_{a}^{2}(t) \hat{g}_{a}^{\dagger} \hat{g}_{a}+\Omega_{b}^{2}(t) \hat{g}_{b}^{\dagger} \hat{g}_{b},
$$

which acts at the subspace of the upper atomic level $c$ (note that $\hat{g}_{a}^{\dagger} \hat{g}_{b}=0$, $\left.\hat{g}_{b}^{\dagger} \hat{g}_{a}=0\right)$. The eigenvectors of this operator form an orthonormal set, while its eigenvalues are real and non-negative. The eigenvectors $\mid D_{n}^{c}(t)>$ with zero eigenvalues:

$$
\hat{G}^{\dagger}(t) \hat{G}(t) \mid D_{n}^{c}(t)>=0, n=1, \ldots, N_{c}^{d},
$$

uncoupled to the lower levels $a$ and $b$, are at the same time the eigenvectors of the interaction operator $\hat{V}(t)$ with the eigenvalues $\lambda_{c n}=-2 \Delta$. The eigenvectors $\mid C_{n}(t)>$ with positive eigenvalues $c_{n}^{2}(t)>0$ :

$$
\hat{G}^{\dagger}(t) \hat{G}(t)\left|C_{n}(t)>=c_{n}^{2}(t)\right| C_{n}(t)>, n=1, \ldots, N_{c},
$$


are coupled by the electric dipole transitions to the states

$$
\left|F_{n}(t)>=\frac{1}{c_{n}(t)} \hat{G}(t)\right| C_{n}(t)>.
$$

These states $\mid F_{n}(t)>$ also form an orthonormal subset at the subspace of the lower atomic levels $a$ and $b$, as it follows from (10)-(11).

With the introduction of the states $\mid C_{n}(t)>$ and $\mid F_{n}(t)>$ all the eigenvectors of the interaction operator $\hat{V}(t)$ with non-zero eigenvalues may be easily obtained. This operator has $2 N_{c}$ eigenvectors

$$
\begin{gathered}
\left|V_{n}^{(+)}(t)>=\sin \theta_{n}(t)\right| F_{n}(t)+\cos \theta_{n}(t) \mid C_{n}(t)>, \\
\left|V_{n}^{(-)}(t)>=\cos \theta_{n}(t)\right| F_{n}(t)>-\sin \theta_{n}(t) \mid C_{n}(t)>, \\
\tan 2 \theta_{n}(t)=-\frac{c_{n}(t)}{\Delta}
\end{gathered}
$$

with the eigenvalues

$$
\lambda_{n}^{( \pm)}(t)=-\Delta \pm \sqrt{\Delta^{2}+c_{n}^{2}(t)}
$$

All the states $\mid D_{n}^{c}(t)>$ and $\mid V_{n}^{( \pm)}(t)>$ constitute the orthonormal set of $N^{f}=$ $2\left(2 J_{c}+1\right)-N_{c}^{d}$ eigenvectors of the operator $\hat{V}(t)$ with non-zero eigenvalues.

The other $N^{d}=N-N^{f}$ eigenvectors $\mid D_{n}(t)>$ of the interaction operator $\hat{V}(t)$ obtain zero eigenvalues. These states - dark states - with the atomic part belonging to the subspace of the lower atomic levels $a$ and $b$ remain uncoupled to the upper level $c$ :

$$
\hat{G}^{\dagger}(t) \mid D_{n}(t)>=0, n=1, \ldots, N^{d} .
$$

As it follows from (11) and (16), all the dark states $\mid D_{n}(t)>$ are orthogonal to all the bright states $\mid F_{n}(t)>$.

Among all the dark states let us distinguish first the dark states $\mid D_{n}^{a}>$ and $\left|D_{n}^{b}\right\rangle$, which atomic part belongs to the lower level $a$ or $b$ only. These states are time independent and may be obtained as the eigenvectors of operators $\hat{g}_{a} \hat{g}_{a}^{\dagger}$ or $\hat{g}_{b} \hat{g}_{b}^{\dagger}$ with zero eigenvalues:

$$
\begin{aligned}
& \hat{g}_{a} \hat{g}_{a}^{\dagger} \mid D_{n}^{a}>=0, k=1, \ldots, N_{a}^{d}, \\
& \hat{g}_{b} \hat{g}_{b}^{\dagger} \mid D_{n}^{b}>=0, k=1, \ldots, N_{b}^{d} .
\end{aligned}
$$

The other $N_{a b}^{d}$ eigenvectors $\mid D_{n}^{a b}(t)>$ of the interaction operator $\hat{V}(t)$, which atomic part belongs to both atomic lower levels $a$ and $b$, obtain zero eigenvalues and satisfy the equation (16), while

$$
\hat{g}_{a}^{\dagger}\left|D_{n}^{a b}(t)>\neq 0, \hat{g}_{b}^{\dagger}\right| D_{n}^{a b}(t)>\neq 0
$$

The temporal dependence of these dark states may be immediately obtained from the equation (16):

$$
\mid D_{n}^{a b}(t)>=Z_{n}(t)\left[\Omega_{a}(t)\left|\tilde{B}_{n}^{d}>-\Omega_{b}(t)\right| A_{n}^{d}>\right],
$$


where $Z_{n}(t)$ is the normalization factor, while $\left|A_{n}^{d}\right\rangle$ and $\left|\tilde{B}_{n}^{d}\right\rangle$ are temporally independent states, which atomic parts belong to the levels $a$ and $b$ correspondingly and which satisfy the equation

$$
\hat{g}_{a}^{\dagger}\left|A_{n}^{d}>=\hat{g}_{b}^{\dagger}\right| \tilde{B}_{n}^{d}>\neq 0 .
$$

Introducing the matrix

$$
\hat{D}_{b}=\sum_{n} \frac{1}{b_{n}^{2}}\left|B_{n}><B_{n}\right|
$$

containing only the eigenvectors $\mid B_{n}>$ of matrix $\hat{g}_{b} \hat{g}_{b}^{\dagger}$ with non-zero eigenvalues $b_{n}^{2}$, we may write the equation (19) as follows:

$$
\left|\tilde{B}_{n}^{d}>=\hat{D}_{b a}\right| A_{n}^{d}>, \hat{D}_{b a}=\hat{D}_{b} \hat{g}_{b} \hat{g}_{a}^{\dagger} .
$$

Now we may define the orthonormal set of states $\left|A_{n}^{d}\right\rangle$ as the eigenvectors of the hermitian matrix $\hat{D}_{b a}^{\dagger} \hat{D}_{b a}$ with non-zero eigenvalues:

$$
\hat{D}_{b a}^{\dagger} \hat{D}_{b a}\left|A_{n}^{d}>=a_{d n}^{2}\right| A_{n}^{d}>, a_{d n}>0 .
$$

Then, as it follows from (20) and (21), the states

$$
\left|B_{n}^{d}>=\frac{1}{a_{d n}} \hat{D}_{b a}\right| A_{n}^{d}>
$$

also constitute the orthonormal set, so that the orthonormal set of dark states $\mid D_{n}^{a b}(t)>$ may be expressed through these states $\mid A_{n}^{d}>$ and $\mid B_{n}^{d}>$ by the formula:

$$
\mid D_{n}^{a b}(t)>=\frac{a_{d n} \Omega_{a}(t)\left|B_{n}^{d}>-\Omega_{b}(t)\right| A_{n}^{d}>}{\sqrt{\Omega_{b}^{2}(t)+a_{d n}^{2} \Omega_{a}^{2}(t)}} .
$$

All the eigenvectors of the interaction operator $\hat{V}(t)$, comprising the set of states $\left|D_{n}^{c}(t)>,\right| V_{n}^{( \pm)}(t)>$, and the set of dark states $\left|D_{n}^{a}>,\right| D_{n}^{b}>$ and $\mid D_{n}^{a b}(t)>$ constitute the complete orthonormal set of states, which determines the evolution operator (7) in the adiabatic approximation.

\section{$3 \quad$ Photon storage and retrieval}

Initially the atom is at the lower level $b$, its state being defined by the atomic density matrix $\hat{\rho}_{0}^{b}$, while the state of the field is a pure single-photon state

$$
\left|f>=\xi_{1}\right| 1,0>+\left.\xi_{2}|0,1>,| \xi_{1}\right|^{2}+\left|\xi_{2}\right|^{2}=1,
$$

where the two complex numbers $\xi_{1}$ and $\xi_{2}$ define the photon polarization (the polarization q-bit), so that the initial density matrix $\hat{\rho}(0)$ of the atom+field system is:

$$
\hat{\rho}(0)=\hat{\rho}_{0}^{b} \cdot|f><f| .
$$


The classical coherent laser field is adiabatically switched off, while the interaction with the quantum field is adiabatically switched on in the time interval $T_{1}$, so that:

$$
\Omega_{a}(0)=\Omega_{a 1}, \Omega_{a}\left(T_{1}\right)=0, \Omega_{b}(0)=0, \Omega_{b}\left(T_{1}\right)=\Omega_{b 1} .
$$

The atomic part of the initial bright states $\mid F_{n}(0)>$, defined by the equation (11), with non-zero eigenvalues $c_{n}^{2}(0) \neq 0$ belongs to the level $a$ only, so that the corresponding initial eigenvectors $\mid V_{n}^{ \pm}(0)>$ of the interaction operator, defined by (12)-(14), will not contribute to the evolution. However the atomic part of the initial bright states $\mid F_{n}(0)>$ with zero eigenvalues $c_{n}^{2}(0)=0$, obtained as the limit at $t \rightarrow+0$, belongs to the level $b$. Since $c_{n}^{2}(0)=\Omega_{a 1}^{2} c_{a n}^{2}$, where $c_{a n}^{2}$ are the eigenvalues of the operator $\hat{g}_{a}^{\dagger} \hat{g}_{a}$, such states will be coupled to the eigenvectors $\mid C_{n}^{b}>$ of the operator $\hat{g}_{a}^{\dagger} \hat{g}_{a}$ with zero eigenvalues:

$$
\hat{g}_{a}^{\dagger} \hat{g}_{a} \mid C_{n}^{b}>=0, n=1, \ldots, N_{c}^{b} .
$$

So, the evolution operator (7) will be determined by the normalized states $\hat{g}_{b} \mid C_{n}^{b}>$ and the dark states $\mid D_{n}^{b}>$ and $\mid D_{n}^{a b}(t)>$, defined by (18) and (23), which atomic part also belongs to the level $b$. The states $\mid D_{n}^{b}>$ in the evolution operator are responsible for the photon passage through the cavity without interaction, while the states $\hat{g}_{b} \mid C_{n}^{b}>$ are responsible for the process of photon absorption and the adiabatic transfer of the atom from the initial level $b$ to the excited level $c$ to some state, which is uncoupled to the lower level $a$. In both cases the final system state is unstable and for purposes of long-term photon storage the presence of such states in the initial system state should be avoided by the proper choice of polarization of the driving field and by the preparation of the atomic state. With such states excluded the evolution is determined only by the states $\mid D_{n}^{a b}(0)>$, which are responsible for the long-term recording of the photon polarization q-bit onto the superposition of Zeeman substates of the metastable level $a$. The evolution operator at the instant of time $t=T_{1}$ after the recording process is finished is as follows:

$$
\hat{S}_{1}=-\sum_{n}\left|A_{n}^{d}><B_{n}^{d}\right|,
$$

so that each state $\left|B_{n}^{d}\right\rangle$ from the level $b$, defined by (22), is adiabatically transferred to the corresponding state $\mid A_{n}^{d}>$ from the level $a$, defined by (21). The total probability $w$ of the photon storage at the level $a$ generally depends on the photon polarization $\xi_{1}$ and $\xi_{2}$ :

$$
w=\operatorname{tr}\left\{\hat{S}_{1} \hat{\rho}(0) \hat{S}_{1}^{\dagger}\right\}=\sum_{i, j=1}^{2} \xi_{i} \xi_{j}^{*} w_{i j}
$$

where

$$
w_{i j}=\sum_{n}<b_{n}^{d i}\left|\hat{\rho}_{0}^{b}\right| b_{n}^{d j}>
$$

while $\left|b_{n}^{d 1}>=<1,0\right| B_{n}^{d}>$ and $\left|b_{n}^{d 2}>=<0,1\right| B_{n}^{d}>$ are the pure atomic states at the level $b$ - the projections of the field+atom states $\mid B_{n}^{d}>$ on the two photon 
polarization states $\mid 1,0>$ and $\mid 0,1>$. For purposes of q-bit storage the total probability must be unity and must not depend on photon polarization, so that the condition

$$
w_{i j}=\delta_{i j}
$$

must be satisfied.

To retrieve the photon after the storage time $\tau$ the classical coherent laser field with the same polarization $\mathbf{l}_{c}$, as that of the field applied to store the photon, is adiabatically switched on, while the interaction with the quantum field is adiabatically switched off in the time interval $T_{2}$, so that:

$$
\begin{gathered}
\Omega_{a}\left(T_{1}+\tau\right)=0, \Omega_{a}\left(T_{1}+\tau+T_{2}\right)=\Omega_{a 2}, \\
\Omega_{b}\left(T_{1}+\tau\right)=\Omega_{b 2}, \Omega_{b}\left(T_{1}+\tau+T_{2}\right)=0 .
\end{gathered}
$$

If the photon was stored with unit probability, then at the start of storage process the atom was prepared at some state of the level $b$, which contained only the parts $\mid B_{n}^{d}>$ of the "dark" states $\left|D_{n}^{a b}\right\rangle$, while the the initially unpopulated level $a$ was populated during the storage process by the adiabatic transfer of the parts $\left|B_{n}^{d}\right\rangle$ of the "dark" states $\mid D_{n}^{a b}>$ to their counterparts $\left|A_{n}^{d}\right\rangle$ at the level $a$, so that at the start of the retrieval stage only the "dark" states $\mid D_{n}^{a b}>$ will be present in the initial atomic state, provided that the polarization of the retrieving field is the same as that of the recording field, ensuring that the "dark" states $\mid D_{n}^{a b}>$ are the same for both stages - the storage and the retrieval. In this case only the dark states $\mid D_{n}^{a b}(t)>$ will contribute to the evolution operator (7). Then, at the end of the retrieval process at the instant of time $t_{2}=T_{1}+\tau+T_{2}$ the evolution operator

$$
\hat{S}_{2}=-\sum_{n}\left|B_{n}^{d}><A_{n}^{d}\right|
$$

is determined by the same states $\mid A_{n}^{d}>$ and $\mid B_{n}^{d}>$ as the evolution operator $\hat{S}_{1}$ (25) and the product of the evolution operators $\hat{S}_{2} \hat{S}_{1}$ is reduced to the projector on the subspace of states $\mid B_{n}^{d}>$ :

$$
\hat{S}_{2} \hat{S}_{1}=\sum_{n}\left|B_{n}^{d}><B_{n}^{d}\right|
$$

and since only such states are present at the initial system state $\hat{\rho}(0)$, then this state will be retrieved without distortion

$$
\hat{\rho}_{2}=\hat{S}_{2} \hat{S}_{1} \hat{\rho}(0) \hat{S}_{2}^{\dagger} \hat{S}_{1}^{\dagger}=\hat{\rho}(0),
$$

reproducing a single photon $|f\rangle$ with the recorded polarization and the atom at the initial state with the density matrix $\hat{\rho}_{0}^{b}$.

Let us choose the two unit polarization vectors $\mathbf{l}_{1}$ and $\mathbf{l}_{2}$ of the quantum field in the plane $X Y$ as the two vectors with opposite circular components:

$$
l_{1 q}=\delta_{q,-1}, l_{2 q}=\delta_{q, 1},
$$


while the polarization vector $\mathbf{l}_{c}$ of the classical field generally contains also $Z$ projections.

In the experiment [5] the angular momenta of the atomic levels were $J_{a}=2$, $J_{b}=J_{c}=1$, while the driving field was linearly polarized along the axis $Z$ : $l_{c q}=\delta_{q, 0}(\pi$-polarized field $)$. In this case there are three states $\mid A_{n}^{d}>$ at the level $a$, defined by (21):

$$
\left|A_{1}^{d}>=\left(\begin{array}{l}
0 \\
0 \\
0 \\
1 \\
0
\end{array}\right),\right| A_{2}^{d}>=\left(\begin{array}{l}
0 \\
1 \\
0 \\
0 \\
0
\end{array}\right), \mid A_{3}^{d}>=\left(\begin{array}{l}
0 \\
0 \\
1 \\
0 \\
0
\end{array}\right),
$$

which are adiabatically coupled to the three states $\mid B_{n}^{d}>$ at the level $b$, defined by (22):

$$
\begin{gathered}
\left|B_{1}^{d}>=\left(\begin{array}{l}
0 \\
1 \\
0
\end{array}\right)\right| 1,0>,\left|B_{2}^{d}>=-\left(\begin{array}{l}
0 \\
1 \\
0
\end{array}\right)\right| 0,1>, \\
\mid B_{3}^{d}>=\frac{1}{\sqrt{2}}\left\{\left(\begin{array}{l}
1 \\
0 \\
0
\end{array}\right)\left|1,0>-\left(\begin{array}{l}
0 \\
0 \\
1
\end{array}\right)\right| 0,1>\right\} .
\end{gathered}
$$

The elements of matrix $w_{i, j}$ (27) determining the photon storage probability are expressed through the elements $\rho_{m_{b}, m_{b}^{\prime}}^{b b}$ of the initial atomic density matrix $\hat{\rho}_{0}^{b}$ in a following way:

$$
\begin{gathered}
w_{1,1}=\rho_{0,0}^{b b}+\frac{1}{2} \rho_{-1,-1}^{b b}, \\
w_{2,2}=\rho_{0,0}^{b b}+\frac{1}{2} \rho_{1,1}^{b b}, \\
w_{2,1}=w_{1,2}^{*}=-\frac{1}{2} \rho_{1,-1}^{b b},
\end{gathered}
$$

so that the conditions (28) may be met only if the atom is initially in the pure state $\mid J_{b}=1, m_{b}=0>$, as it was in the experiment. Then, as it follows from the expression (25) for the evolution operator, the initial system pure state

$$
\mid J_{b}=1, m_{b}=0>\left(\xi_{1}\left|1,0>+\xi_{2}\right| 0,1>\right)
$$

is adiabatically transferred to the pure state

$$
\left(\xi_{2}\left|J_{a}=2, m_{a}=-1>-\xi_{1}\right| J_{a}=2, m_{a}=1>\right) \mid 0,0>.
$$

The conditions (28) may be also met, as calculations show, with the driving field linearly polarized along the axis $X$ :

$$
l_{c q}=\frac{1}{\sqrt{2}}\left(\delta_{q,-1}-\delta_{q, 1}\right)
$$


and with the same initial atomic state $\mid J_{b}=1, m_{b}=0>$, though in this case the photon polarization will be mapped to the superposition of states $\mid J_{a}=$ $2, m_{a}=0>$ and $\mid J_{a}=2, m_{a}= \pm 2>$ :

$$
\begin{aligned}
& a^{0}\left|m_{a}=0>+a^{+}\right| m_{a}=2>+a^{-} \mid m_{a}=-2>, \\
& a^{0}=\frac{\xi_{1}+\xi_{2}}{2 \sqrt{2}}, a^{ \pm}=\frac{\sqrt{3}\left(\xi_{1}+\xi_{2}\right)}{4} \pm \frac{\left(\xi_{1}-\xi_{2}\right)}{2} .
\end{aligned}
$$

For the lower values $J_{b}=0$ and $J_{b}=1 / 2$ of the angular momenta of the initial atomic level $b$ the conditions (28) for the photon storage with unit probability, independent on photon polarization, may be easily met. With the driving field linearly polarized along the axis $Z: l_{c q}=\delta_{q, 0}$, these conditions will be satisfied, for example, for the transitions with $J_{b}=0, J_{a}=J_{c}=1$ and with $J_{b}=1 / 2$, $J_{a}=J_{c}=3 / 2$ for any initial atomic state, in particular, for the equilibrium state: $\rho_{-1 / 2,-1 / 2}^{b b}=\rho_{1 / 2,1 / 2}^{b b}=1 / 2, \rho_{-1 / 2,1 / 2}^{b b}=\rho_{1 / 2,-1 / 2}^{b b}=0$. However for larger values of angular momentum $J_{b}>1$ it becomes hard (if not impossible) to meet the conditions (28). Indeed, the memory efficiency (or better to say inefficiency) is determined by the number $N_{d}^{b}$ of the "dark" states of the type $\mid D_{n}^{b}>$, defined by the equation (18), and by the weight of these states in the initial atomic state. The number of states $2\left(2 J_{b}+1\right)$ with the atomic part at the level $b$ is doubled due to the two possible polarizations of the cavity field and only $2 J_{c}+1$ of them are coupled to the excited level $c$, while the other $N_{d}^{b}=2\left(2 J_{b}-J_{c}+1\right)-1$ states - the "dark" states of the type $\mid D_{n}^{b}>-$ stay uncoupled, reducing the memory efficiency. The number of such states obtains its minimum $N_{d}^{b}=2 J_{b}-1$ on the transitions $J_{b} \rightarrow J_{c}=J_{b}+1$, so that at $J_{b} \geq 1$ the presence of these states becomes mandatory. The number $N_{d}^{b}$ of the states $\mid D_{n}^{b}>$ grows with $J_{b}$ and at $J_{b}>1$ it becomes highly improbable that each Zeeman state $\mid J_{b}, m_{b}>$ will not be contained in these "dark" states $\mid D_{n}^{b}>$. The general approach, developed in the present paper, enables one to analyze numerically the memory efficiency for any reasonable values of the angular momenta of resonant atomic levels. For example, let us consider the transitions $J_{b}=2 \rightarrow J_{c}=3 \rightarrow J_{a}=4$ and the $\pi$-polarized driving field $\left(l_{c q}=\delta_{q, 0}\right)$. Then the elements of the probability matrix $w_{i, j}$ (27) are expressed through the elements $\rho_{m_{b}, m_{b}^{\prime}}^{b b}$ of the initial atomic density matrix $\hat{\rho}_{0}^{b}$ in a following way:

$$
\begin{aligned}
& w_{1,1}=\frac{1}{7} \rho_{-2,-2}^{b b}+\frac{1}{2} \rho_{-1,-1}^{b b}+\frac{6}{7} \rho_{0,0}^{b b}+\rho_{1,1}^{b b}+\rho_{2,2}^{b b}, \\
& w_{2,2}=\frac{1}{7} \rho_{2,2}^{b b}+\frac{1}{2} \rho_{1,1}^{b b}+\frac{6}{7} \rho_{0,0}^{b b}+\rho_{-1,-1}^{b b}+\rho_{-2,-2}^{b b},
\end{aligned}
$$

while $w_{1,2}$ and $w_{2,1}$ are expressed only through the non-diagonal elements $\rho_{m_{b}, m_{b}^{\prime}}^{b b}$ $\left(m_{b} \neq m_{b}^{\prime}\right)$ of the atomic density matrix. In addition the populations of Zeeman sublevels are non-negative $\rho_{m m}^{b b} \geq 0$ and the total population of the level is unity

$$
\rho_{-2,-2}^{b b}+\rho_{-1,-1}^{b b}+\rho_{0,0}^{b b}+\rho_{1,1}^{b b}+\rho_{2,2}^{b b}=1 .
$$


For a hundred per cent memory efficiency $w_{1,1}=w_{2,2}=1$, then extracting (31) from (33) we obtain

$$
\frac{6}{7} \rho_{-2,-2}^{b b}+\frac{1}{2} \rho_{-1,-1}^{b b}+\frac{1}{7} \rho_{0,0}^{b b}=0
$$

which is true only if $\rho_{-2,-2}^{b b}=\rho_{-1,-1}^{b b}=\rho_{0,0}^{b b}=0$, and extracting (32) from (33) we also obtain that $\rho_{2,2}^{b b}=\rho_{1,1}^{b b}=0$, so that in this case the photon q-bit cannot be stored with unit probability. However, if the atom is initially prepared at the state $\mid J_{b}=2, m_{b}=0>$, then $w_{i j}=(6 / 7) \delta_{i j}$, and the photon will be stored with the probability $w=6 / 7$ independent of its polarization.

\section{Conclusions}

In the present paper we have obtained the general expression for the probability of long-term storage of a single-photon polarization q-bit at the magnetic substates of some long-lived degenerate atomic level by means of STIRAP. The dependence of this probability on the polarization of the driving field and on the initial atomic state was studied. It was shown that the conditions for the photon long-term storage with unit probability independent on the photon polarization may be satisfied with low values of angular momentum of the initial atomic level $J_{b}=0,1 / 2,1$. At larger values of this angular momentum $J_{b}>1$ it becomes hard (if not impossible) to store the arbitrarily polarized photon with unit probability. The general approach, developed in the present paper, enables one to analyze numerically the memory efficiency for any reasonable values of the angular momenta of resonant atomic levels. It was also shown that, since the conditions of the photon storage with unit probability are satisfied, the driving field with the same polarization as that used to store the photon will retrieve the initial atomic state and a single photon with the recorded polarization without distortion.

Acknowledgements

Authors are indebted for financial support of this work to Russian Ministry of Science and Education (grant 2407).

\section{References}

[1] Lvovsky A, Sanders B and Tittel W 2009 Nature Photonics 3706

[2] Simon C et al 2010 Eur. Phys. J. D 581

[3] Kimble H 2008 Nature 4531023

[4] Simon C, Riedmatten H and Gisin N 2011 Rev.Mod.Phys. 8333

[5] Specht H et al 2011 Nature 473190

[6] Riedl S et al 2012 Phys. Rev. A 85022318 
[7] Himsworth M et al 2011 Appl.Phys. B 103579

[8] Viscor D et al 2011 J. Phys. B: At. Mol. Opt. Phys. 44195504

[9] Hennrich M, Legero T, Kuhn A and Rempe G 2000 Phys.Rev.Lett. 854872

[10] Kuhn A, Hennrich M and Rempe G 2002 Phys.Rev.Lett. 89067901

[11] Wilk T et al 2007 Phys.Rev.Lett. 98063601

[12] Fleischhauer M, Imamoglu A and Marangos J 2005 Rev.Mod.Phys. 77633

[13] Scully M and Zubairy M 1997 Quantum Optics (Cambridge: Cambridge University Press)

[14] Kis Z, Karpati A, Shore B and Vitanov N 2004 Phys.Rev. A 70053405

[15] Sobelman I 1972 Introduction to the Theory of Atomic Spectra (New York: Pergamon) 